\title{
AABEAM SPECTRAL FINITE ELEMENTS FOR MODELLING OF PROPAGATING WAVES IN COMPOSITE LAMINATES UNDER ENVIRONMENTAL EFFECTS
}

\author{
W. Ostachowicz ${ }^{1,3}$, P. Kudela ${ }^{1,2}$, K. Lonkar $^{2}$ \\ ${ }^{1}$ Department of Mechanics of Intelligent Structures, Polish Academy of Sciences \\ (wieslaw@imp.gda.pl)
}

${ }^{2}$ Structures and Composites Laboratory, Stanford University

${ }^{3}$ Faculty of Navigation, Gdynia Maritime University

\begin{abstract}
This paper present a numerical model based on Timoshenko theory and spectral element method. The model is used for wave propagation analysis under environmental effects such as external loading, temperature and moisture. The model also incorporates open crack calculated using fracture mechanics laws. The aim of this paper is investigation of various features extensively used in application for damage detection such as wave packet amplitude, time of flight or peak of power spectral density. The study of these parameters is necessary for compensation of environmental influence on wave propagation which affects accuracy of damage identification.
\end{abstract}

Keywords: Spectral Element Method, wave propagation, environmental effects, composites.

\section{INTRODUCTION}

Wave propagation phenomenon is extensively used in the field of non-destructive testing and more recently in the field of Structural Health Monitoring (SHM). One of the axioms of SHM is that the assessment of damage requires a comparison between two system states. In an ideal case the difference between damage state and healthy state of wave propagation signals gives full information about the damage. But wave propagation signals are also affected by the environmental effects such as temperature, moisture and initial stresses. In such a case raw signal subtraction between two system states could lead to false alarms or misclassification of damage type or wrong estimation of the size of the damage. For this reason it is necessary to develop compensation methods which would be able to discard environmental effects and extract information about damage only.

The problem described above is very complex and cannot be solved analytically. Only few research papers could be found dealing with the influence of environmental effects on propagation of elastic waves.

Composite structures exposed to extreme temperatures may lead to damage in the form of debonding especially in sandwich structures. Problem of diagnosis of such defects 
was addressed by Blaise and Chang [1]. Many damage detection systems are based on a network of piezoelectric sensors and utilise anomalies in Lamb wave propagation. However, anomalies in Lamb wave propagation come from not only damage but also from changes in temperatures. The most visible influence of temperature on propagating waves is in velocity which in turn leads to inaccurate damage localisation. This effect is directly correlated to thermal expansion of material and changes in Young's modulus. It has been shown that for isotropic materials there is linear relation between temperature and time delay of arriving wave packets of propagating waves [2]. The effect of time delay can be compensated by two methods: optimal baseline stretch or optimal baseline subtraction reported in [3]. It should be pointed out that the temperature influences not only velocity of propagating waves but also their amplitudes. Additionally this effect is no more linear. Hence more advanced method for temperature compensation should be applied. Such a method can be based on mechanical model and machine learning algorithm [4]. Another issue which should be mentioned is adhesive layer property between piezoelectric transducer and structure. The adhesive shear modulus is most influential parameter considering amplitudes of propagating waves. It has been shown in [5] that decrease in adhesive shear modulus at a higher temperature $\left(75^{\circ} \mathrm{C}\right)$ causes the amplitude to increase at $350 \mathrm{kHz}$ while it causes the amplitude to decrease at 150 $\mathrm{kHz}$ and $500 \mathrm{kHz}$.

Most of research related to temperature effects on Lamb waves are focus on isotropic structures. Not much attention has been paid to composite materials. In such materials temperature causes degradation of material properties in a nonlinear manner unlike metallic materials such as aluminium and steel alloys. The influence of thermal degradation on local changes in elastic waves propagating in composite laminates has been investigated numerically by Kudela et al. [6]. The effects of temperature in terms of thermal expansion as well as degradation of Young's modulus of a matrix in composite laminate and their influence on propagating waves are investigated in this paper.

Apart from the temperature moisture must be considered. Moisture affects material properties and causes so called swelling of the matrix which is associated with the entrapment of moisture in the polymer matrix resulting in a weight increase $(<2 \%)$ [7]. The relation between moisture content and composite material properties is quite well established [8]. Moreover, research work has been reported on tracking hygrothermal strains of carbon/epoxy composites [9]. However, studies of dynamic behaviour of composite structures under hygrothermal conditions are limited to free vibrations [10].

Another factor influencing propagating waves, especially velocities of elastic waves, is external loading or initial stress field introduced for example during manufacturing process. There are few techniques mentioned in [11] for obtaining dispersion curves which take into account initial stresses. For simple geometries such as plates and pipes there are analytical methods. However, for more complicated cross sections, numerical methods such as finite element modelling must be applied. These include extracting dispersion relation from the time domain signals calculated by the finite element method, low frequency eigensolvers [11] and semi-analytical finite element method [12]. Again these methods have been applied mostly to isotropic structures such as rails. It has been shown that applied stresses to beam-like structures influence phase velocity curves in low frequency regime [11].

Lematre et al. [13] investigated the influence of a prestress gradient on guided wave propagation in piezoelectric structures. They have found that for a piezoelectric plate, the Lamb and shear horizontal modes are sensitive to the prestress gradient. Another interesting work has been published by Chakraborty [14] who studied surface stress and surface elasticity influence on Lamb waves in ultra-thin films. He replaced lateral stress free boundary conditions, as it is in classical Lamb wave definition, with more complicated non-classical 
boundary conditions coupling the bulk and surface stress. As a result changes in phase velocities are observed.

In this paper a spectral finite beam element based on Timoshenko theory is developed which takes into account temperature, moisture, initial stresses and matrix cracking of composite laminate. In case of temperature and moisture both expansion and degradation of elastic constants are included. Initial stresses are applied by calculation of geometric stiffness matrix. Cracking of composite matrix lamina is calculated according to fracture mechanics law. Algorithm for solving equation of motion utilises the central difference scheme.

Study of parameters such as time of flight (TOF), amplitude change and power spectral density of wave propagation signals is performed and illustrated by numerical examples.

\section{NUMERICAL MODEL}

Spectral finite element applied for modelling of composite beam with a transverse crack is based on Timoshenko theory. The scheme of the element is shown in Fig. 1. It consists of two spectral elements with 8 nodes each one. Nodes are non-uniformly distributed and coincide with Gauss-Lobatto-Legendre points. This property distinguishes spectral finite elements over classical finite elements. Two spectral elements shown in Fig. 1 have separated nodes at crack location and are connected by the spring of bending stiffness $k_{b}$ and shear stiffness $k_{s}$.
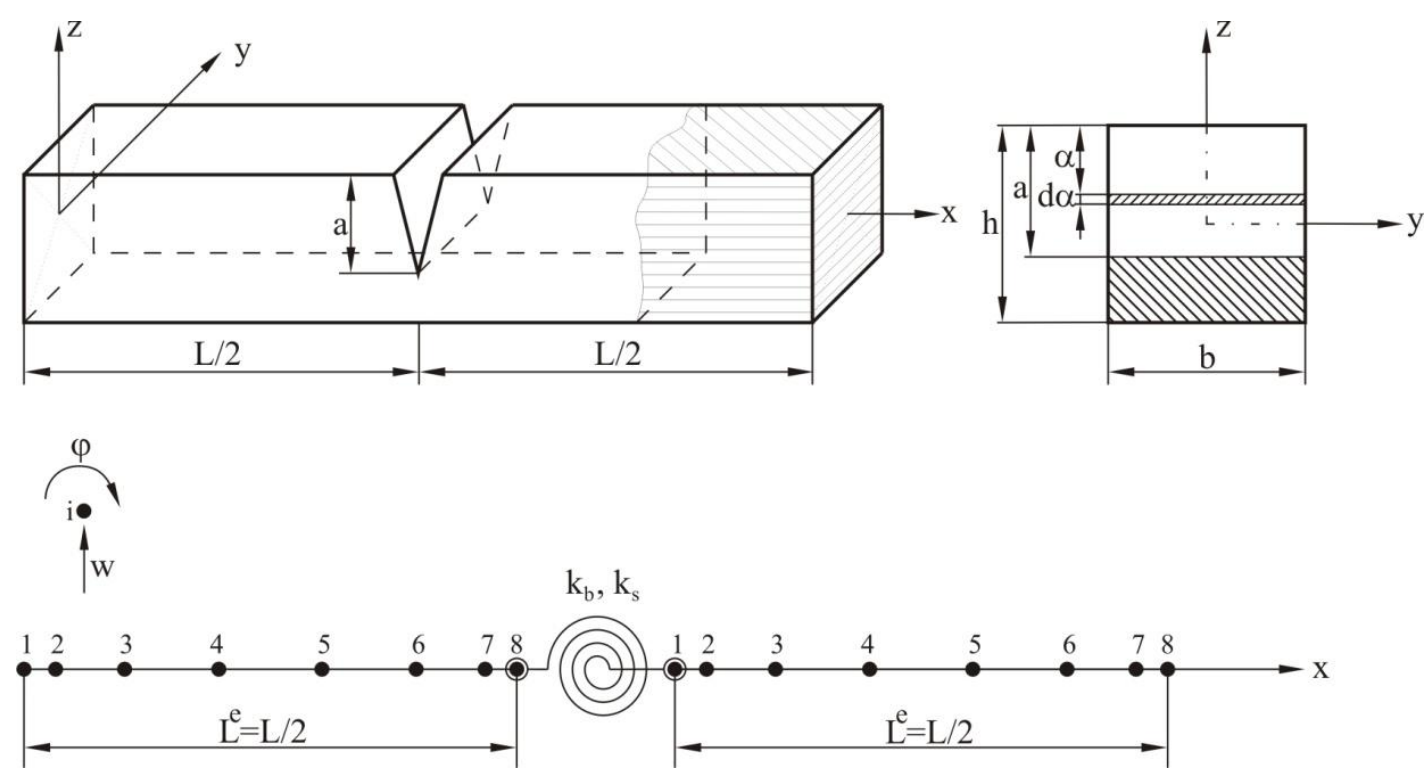

Figure 1. Scheme of the model of composite beam with transverse crack.

The element has two degrees of freedom per node: transverse displacement $w$ and rotation of the cross-section $\varphi$. The displacement field in Timoshenko theory of beams is as follows:

$$
\left\{\begin{array}{l}
u(x, z)=\varphi_{0}(x) \cdot z \\
w(x, z)=w_{0}(x) .
\end{array}\right.
$$


The strain field can be expressed as:

$$
\left\{\begin{array}{l}
\varepsilon_{x}(x, z)=\frac{\partial \varphi_{0}(x)}{\partial x} \cdot z+\varepsilon_{T}+\varepsilon_{M} \\
\gamma_{z x}(x, z)=\frac{\partial w_{0}(x)}{\partial x}+\varphi_{0}(x)
\end{array}\right.
$$

where thermal and hygrothermal strains, $\varepsilon_{T}, \varepsilon_{M}$, are calculated as:

$$
\varepsilon_{T}=-\alpha_{x} \Delta T, \quad \varepsilon_{M}=-\beta_{x} \Delta m
$$

Thermal and moisture expansion coefficients $\alpha_{x}, \beta_{x}$ are calculated based on a rule of mixture and strongly depends on volume fraction of reinforcing fibres [15]. $\Delta T=T-T_{0}$ denotes temperature change with respect to reference temperature $T_{0}$ assumed as $20{ }^{\circ} \mathrm{C}$ in this case. $\Delta m=C-C_{0}$ denotes change in moisture concentration with respect to reference moisture concentration $C_{0}$ assumed as 0 in this case (dry lamina). It should be added that weight gain up to $1.5 \%$ due to moisture absorption is considered.

The approximation of the displacement field can be expressed in the form:

$$
\left\{\begin{array}{l}
w_{0}(\xi) \\
\varphi_{0}(\xi)
\end{array}\right\}=\mathbf{N u}=\sum_{i=1}^{8} N_{i}(\xi) \mathbf{I}\left\{\begin{array}{l}
u_{w}\left(\xi_{i}\right) \\
u_{\varphi}\left(\xi_{i}\right)
\end{array}\right\}, \quad \mathbf{I}=\left[\begin{array}{ll}
1 & 0 \\
0 & 1
\end{array}\right] .
$$

where $\mathbf{N}$ are shape functions and $\mathbf{u}$ are nodal values of degrees of freedom in element. Derivation of characteristic stiffness and mass matrices is performed in a standard manner [16]. Due to consideration of initial stresses also geometric stiffness matrix must be calculated according to the definition:

$$
\mathbf{k}_{g}=\int_{0}^{L} \mathbf{G}^{T} \mathbf{S G} \mathrm{dL}, \quad \mathbf{G}=\frac{\partial \mathbf{N}}{\partial x}
$$

where $\mathbf{S}$ is the matrix of initial stresses:

$$
\mathbf{S}=\left[\begin{array}{cccccc}
\sigma_{x x} & 0 & 0 & 0 & \sigma_{x z} & 0 \\
0 & \sigma_{x x} & 0 & 0 & 0 & \sigma_{x z} \\
0 & 0 & 0 & 0 & 0 & 0 \\
0 & 0 & 0 & 0 & 0 & 0 \\
\sigma_{x z} & 0 & 0 & 0 & 0 & 0 \\
0 & \sigma_{x z} & 0 & 0 & 0 & 0
\end{array}\right]
$$

It should be noted that values of initial stresses might vary across the element and the integration must be performed at Gauss-Lobatto-Legendre points accordingly. 


\subsection{Flexibilities at the crack location}

Coefficients of the beam flexibility matrix at the crack location can be calculated using Castigliano theorem:

$$
c_{i j}=\frac{\partial^{2} U}{\partial F_{i} \partial F_{j}} \quad \text { for } i=j=1 . .2
$$

where $U$ denotes the elastic strain energy of the element by the presence of the crack and $F$ are the independent nodal forces acting on the element. For analyzed beam of rectangular cross-section and transverse open crack along width of the beam, the elastic strain energy due to the crack can be expressed by:

$$
U=\frac{1}{E} \int_{A}\left(K_{I}^{2}+K_{I I}^{2}\right) \mathrm{d} A,
$$

where $A$ denotes the area of the crack, $K_{I}$ and $K_{I I}$ are stress intensity factors corresponding to the first and second mode of the crack growth [17]. The stress intensity factors can be calculated as:

$$
K_{I}=\frac{6 M}{b h^{2}} \sqrt{\pi \alpha} f_{I}\left(\frac{\alpha}{h}\right), \quad K_{I I}=\frac{\beta T}{b h} \sqrt{\pi \alpha} f_{I I}\left(\frac{\alpha}{h}\right)
$$

where $M$ is bending moment, $\beta$ is shear factor, $T$ is shear force, $b, h, \alpha$ are dimensions (see Fig. 1), $f_{I}$ and $f_{I I}$ are correction functions in the form:

$$
\begin{aligned}
& f_{I}\left(\frac{\alpha}{h}\right)=\sqrt{\frac{\tan (\pi \alpha / 2 h)}{\pi \alpha / 2 h}} \frac{0.752+2.02(\alpha / h)+0.37[1-\sin (\pi \alpha / 2 h)]^{3}}{\cos (\pi \alpha / 2 h)} \\
& f_{I I}\left(\frac{\alpha}{h}\right)=\frac{1.30-0.65(\alpha / h)+0.37(\alpha / h)^{2}+0.28(\alpha / h)^{3}}{\sqrt{1-(\alpha / h)}} .
\end{aligned}
$$

After transformation, the bending flexibility and shear flexibility at the crack location between spectral Timoshenko beam elements can be rewritten as:

$$
c_{b}=\frac{72 \pi}{\tilde{E} b h^{2}} \int_{0}^{a / h} \frac{\alpha}{h} f_{I}^{2}\left(\frac{\alpha}{h}\right) \mathrm{d}(a / h), \quad c_{s}=\frac{2 \beta \pi}{\tilde{E} b} \int_{0}^{a / h} \frac{\alpha}{h} f_{I I}^{2}\left(\frac{\alpha}{h}\right) \mathrm{d}(a / h),
$$

where $\tilde{E}$ is Young's modulus averaged over thickness of a composite laminate.

The stiffness at the crack location is an inverse of flexibility. Hence, the stiffness matrix which models a crack and connects two spectral elements has a form: 


$$
\mathbf{k}_{s}=\left[\begin{array}{cccc}
1 / c_{s} & 0 & -1 / c_{s} & 0 \\
0 & 1 / c_{b} & 0 & -1 / c_{b} \\
-1 / c_{s} & 0 & 1 / c_{s} & 0 \\
0 & -1 / c_{b} & 0 & 1 / c_{b}
\end{array}\right]
$$

This matrix is added to the global stiffness matrix at the stage of assembly.

\subsection{The algorithm for wave propagation}

Thermal and hygrothermal strains from Eq. 2 cause expansion of the material or give rise to stresses if such expansion is restricted by boundary conditions. Hence, wave propagation analysis must take into account both initial stresses and applied external load as well as excitation signal.

The dependence of guided wave velocity on applied load is a fundamentally nonlinear effect and must be modelled in a two step finite element calculation. Essentially the first step is to compute the displacement of the model under static load and the second step is to model the wave propagation. Both individual steps are linear, the non-linearity of the complete model is introduced by forcing a recalculation of the global stiffness matrix between the two steps. This means that the wave propagation modelling is undertaken with the global stiffness matrix for the deformed waveguide after loading [11].

In the first step, displacements are calculated from standard linear system of equations:

$$
\mathbf{k} \mathbf{u}=\mathbf{F} \rightarrow \mathbf{u} \rightarrow \boldsymbol{\varepsilon} \rightarrow \boldsymbol{\sigma},
$$

where $\mathbf{F}$ is the vector of external forces and $\mathbf{k}$ is global stiffness matrix. Strains and stresses are evaluated based on displacement field. In the second step of the algorithm geometric stiffness matrix (see Eq. 5) is created. Calculation of the geometric stiffness matrix $\mathbf{k}_{g}$ is carried out based on stresses from Eq. 13 as well as thermal and hygrothermal stresses. Finally equation of motion is obtained:

$$
\mathbf{m} \ddot{\mathbf{u}}+\left[\mathbf{k}+\mathbf{k}_{g}(\sigma)\right] \mathbf{u}=\mathbf{s}(t) .
$$

where $\mathbf{m}$ is the mass matrix and $\mathbf{s}(t)$ is the excitation signal. The global stiffness matrix $\mathbf{k}$ is modified by addition of the geometric stiffness matrix $\mathbf{k}_{g}$. Equation 14 is solved by application of central difference scheme [16].

\section{NUMERICAL SIMULATIONS}

Scheme of the beam under investigation is shown in Fig. 2. The beam is subjected to static load $F$ applied at the left end, various uniform changes in temperature $\Delta T$ as well as in 
moisture concentration $\Delta m$. The beam is excited in the centre by sinusoidal signal modulated by Hanning window. Various carrier frequencies between $20 \mathrm{kHz}$ and $48 \mathrm{kHz}$ were used.

It was assumed that the beam is made out of glass/epoxy composite with stacking sequence $[+45 /-45]_{6}$ and $50 \%$ of volume fraction of glass reinforcing fibres. The width of the beam was $20 \mathrm{~mm}$, and the total thickness was $12 \mathrm{~mm}$. Crack depth equal to $15 \%$ of crosssection height was introduced at distance of $0.73 \mathrm{~m}$ from the left end of the beam. The total length of the beam was $1 \mathrm{~m}$.

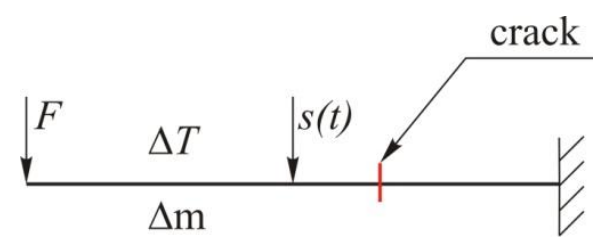

Figure 2. Scheme of the beam under investigation.

Properties of glass/epoxy lamina under various moisture concentrations and various temperatures are given in Table 1 and Table 2, respectively.

Table 1. Elastic moduli of glass/epoxy lamina at various moisture concentrations assumed for calculations, vol $=50 \%$

\begin{tabular}{|c|r|r|r|r|r|r|r|}
\hline Elastic modulus & \multicolumn{7}{|c|}{ Moisture concentration, C $(\%)$} \\
\cline { 2 - 8 }$[\mathrm{GPa}]$ & \multicolumn{1}{|c|}{0.00} & \multicolumn{1}{|c|}{0.25} & \multicolumn{1}{|c|}{0.50} & \multicolumn{1}{|c|}{0.75} & \multicolumn{1}{c|}{1.00} & \multicolumn{1}{c|}{1.25} & \multicolumn{1}{c|}{1.50} \\
\hline$E_{1}$ & 34.96 & 34.92 & 34.87 & 34.83 & 34.80 & 34.78 & 34.78 \\
\hline$E_{2}$ & 9.06 & 8.87 & 8.63 & 8.41 & 8.26 & 8.19 & 8.19 \\
\hline$G_{12}$ & 3.39 & 3.32 & 3.23 & 3.14 & 3.09 & 3.06 & 3.06 \\
\hline
\end{tabular}

Table 1. Elastic moduli of glass/epoxy lamina at various temperatures assumed for calculations, vol $=50 \%$

\begin{tabular}{|c|r|r|r|r|r|r|r|}
\hline \multirow{2}{*}{$\begin{array}{c}\text { Elastic modulus } \\
{[\mathrm{GPa}]}\end{array}$} & \multicolumn{1}{|c|}{ Temperature, T $\left({ }^{\circ} \mathrm{C}\right)$} \\
\cline { 2 - 8 } & \multicolumn{1}{|c|}{20} & \multicolumn{1}{c|}{25} & \multicolumn{1}{c|}{30} & \multicolumn{1}{c|}{35} & \multicolumn{1}{c|}{40} & \multicolumn{1}{c|}{45} & \multicolumn{1}{c|}{50} \\
\hline$E_{1}$ & 34.96 & 34.94 & 34.92 & 34.90 & 34.85 & 34.77 & 34.65 \\
\hline$E_{2}$ & 9.06 & 8.93 & 8.85 & 8.74 & 8.54 & 8.16 & 7.56 \\
\hline$G_{12}$ & 3.39 & 3.34 & 3.31 & 3.27 & 3.19 & 3.05 & 2.82 \\
\hline
\end{tabular}

For each analysed case feature extraction was performed. Three types of features were considered: amplitude, time of flight and peak of power spectra density. These features were calculated from the first incoming wave packet as well as the second wave packet reflected from damage by extracting sampling points from the range of 500 to 1500 and 1700 to 2450, respectively (see Fig. 3). The amplitudes were calculated by finding maximum value of Hilbert transformed signal within ranges described above. Similar procedure was used for calculation of time of flight. Peak value of power spectra density was calculated by using Welch's method. 


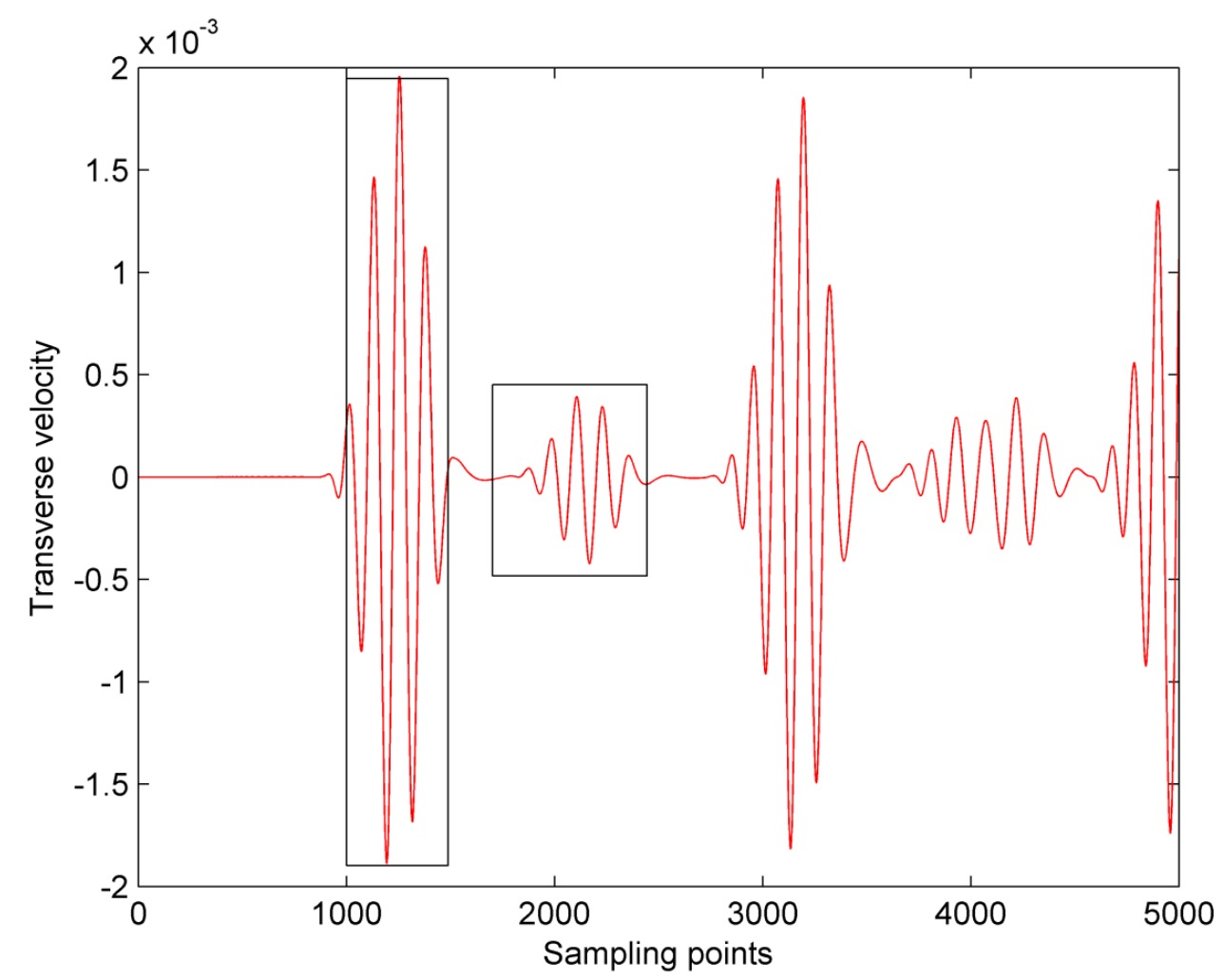

Figure 3. Signal for excitation frequency $20 \mathrm{kHz}$ with indicated wave packets under investigation: incoming wave packet and reflection from the crack

The results of numerical simulations are shown in Fig. 4-6. Figure 4 refers to thermal degradation exclusively. Due to free boundary condition at one of the ends of the beam along with uniform temperature distribution only thermal expansion occurs without any induced thermal stresses. Thermal expansion has minimal effect on wave propagation in comparison to thermal degradation (decrease of the Young's modulus of the epoxy) and is not shown here. It can be seen from Fig. 4 that features dependence on frequency and temperature change is strongly nonlinear. It is interesting to note that by comparing left and right columns of Fig. 4a it is possible to distinguish between incoming wave packet and wave packet reflected from the crack. The same can be said about Fig. 4c which refers to the peak of power spectra density. Time of flight distribution is similar for both wave packets.

Similar effects can be observed due to moisture degradation. Again moisture expansion and mass gain due to moisture absorption much less affects wave propagation than moisture degradation. For this reason only the effect of moisture degradation is shown in Fig. 5. Due to the fact that moisture degradation acts similar as thermal degradation on propagating waves it would be very difficult to separate those two effects.

The influence of transverse load applied on the free end of the beam on wave propagation is much less noticeable than the effects of moisture and temperature (see Fig. 6). It should be pointed out that the selected range of loading conforms to reasonable physical case. It is also important to add that calculations have been carried out on undeformed structure. In such a case both amplitude (Fig. 6a) and time of flight (Fig. 6b) are slightly affected by external loading in a linear manner. 

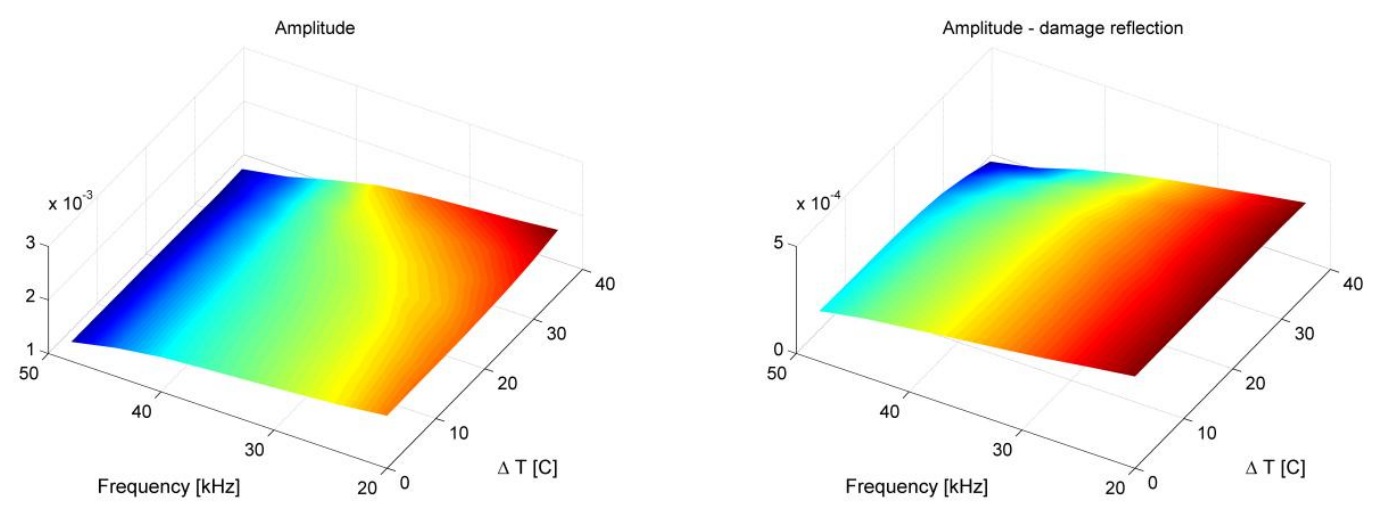

a) Amplitude
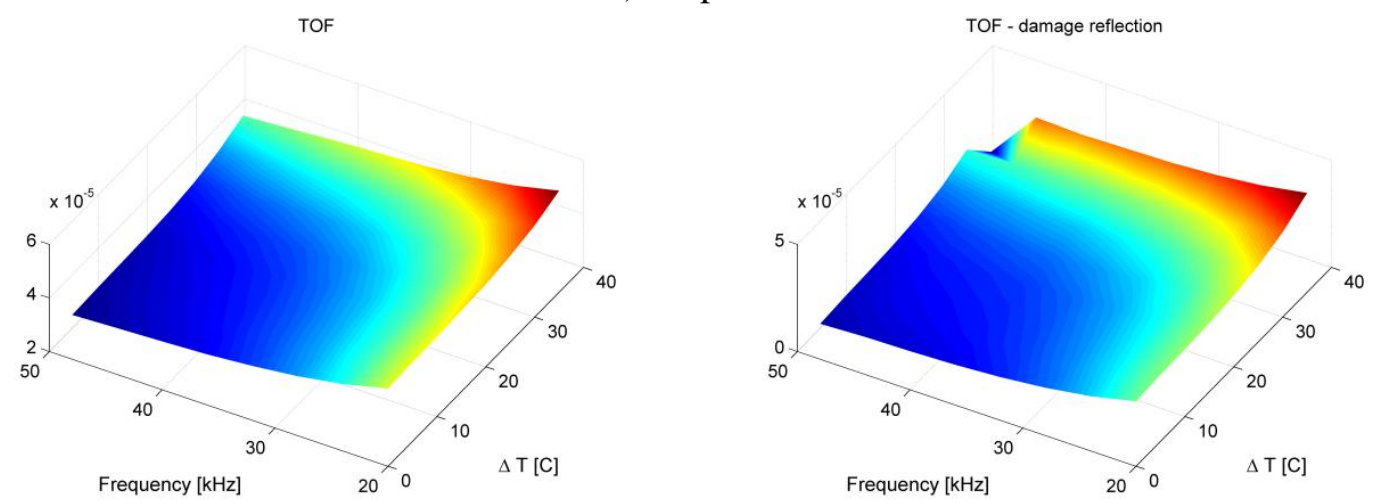

b) Time of Flight (TOF)

PSDP
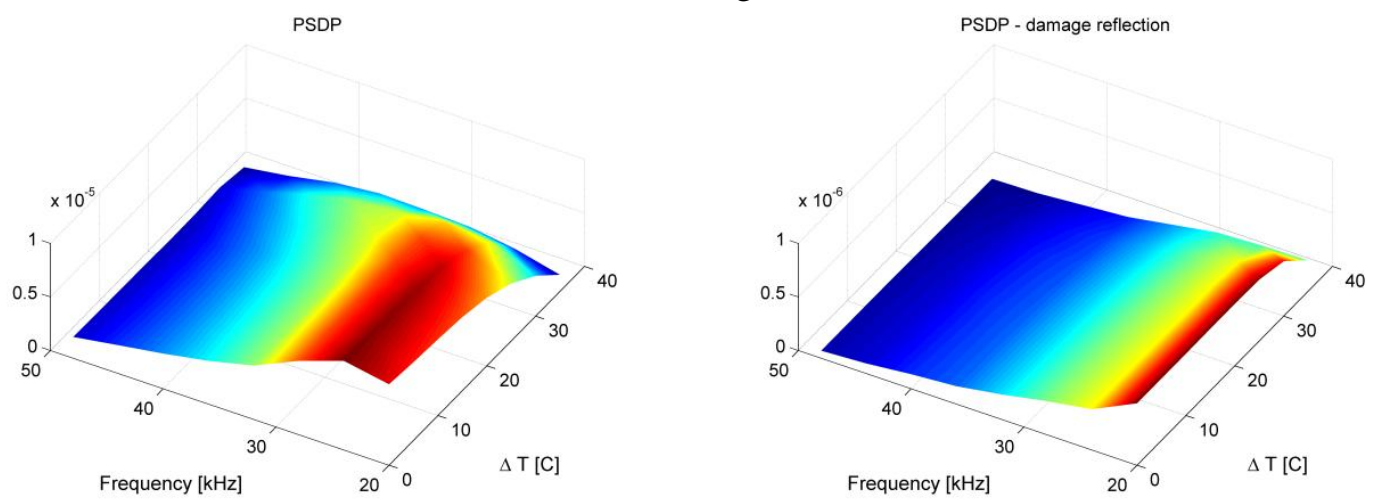

c) Power Spectra Density Peak (PSDP)

Figure 4. Dependence of features on temperature change (thermal degradation). Left column features for the incoming wave packet, right column - features for the wave packet reflected from the crack. 

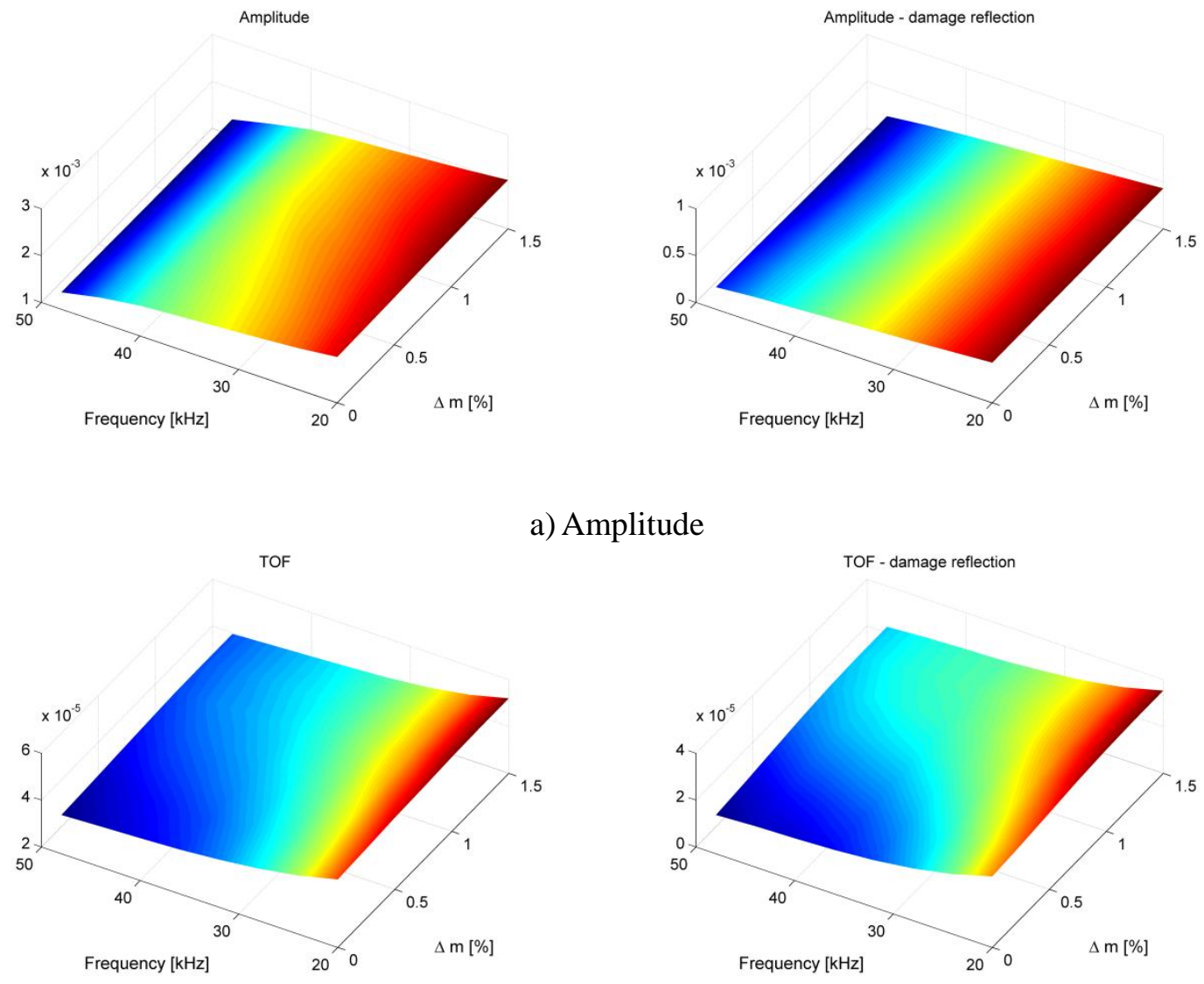

a) Amplitude

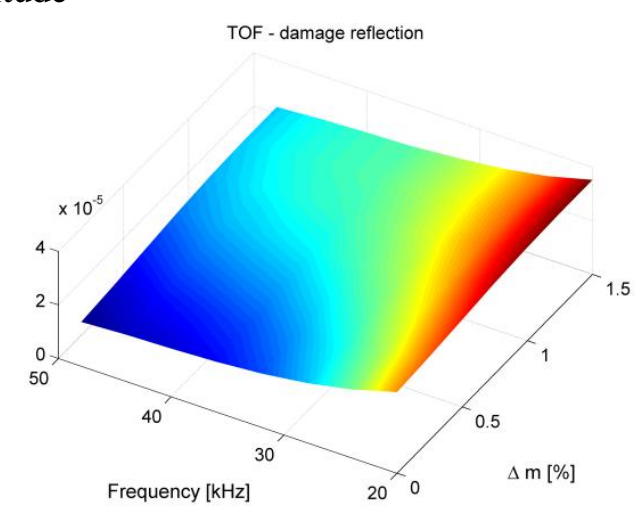

b) Time of Flight (TOF)

PSDP

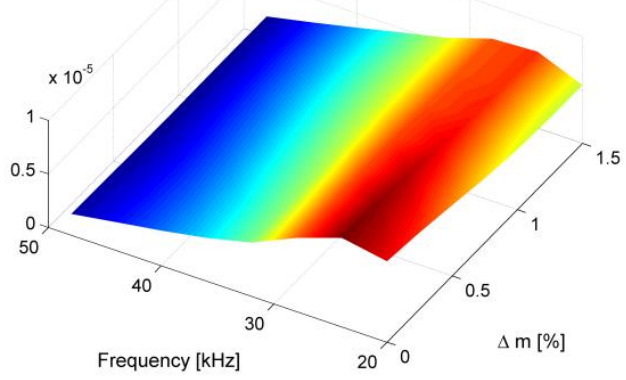

PSDP - damage reflection

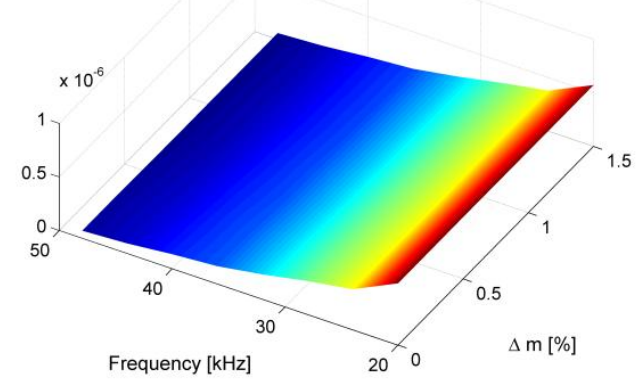

c) Power Spectra Density Peak (PSDP)

Figure 5. Dependence of features on moisture (moisture degradation). Left column - features for the incoming wave packet, right column - features for the wave packet reflected from the crack. 

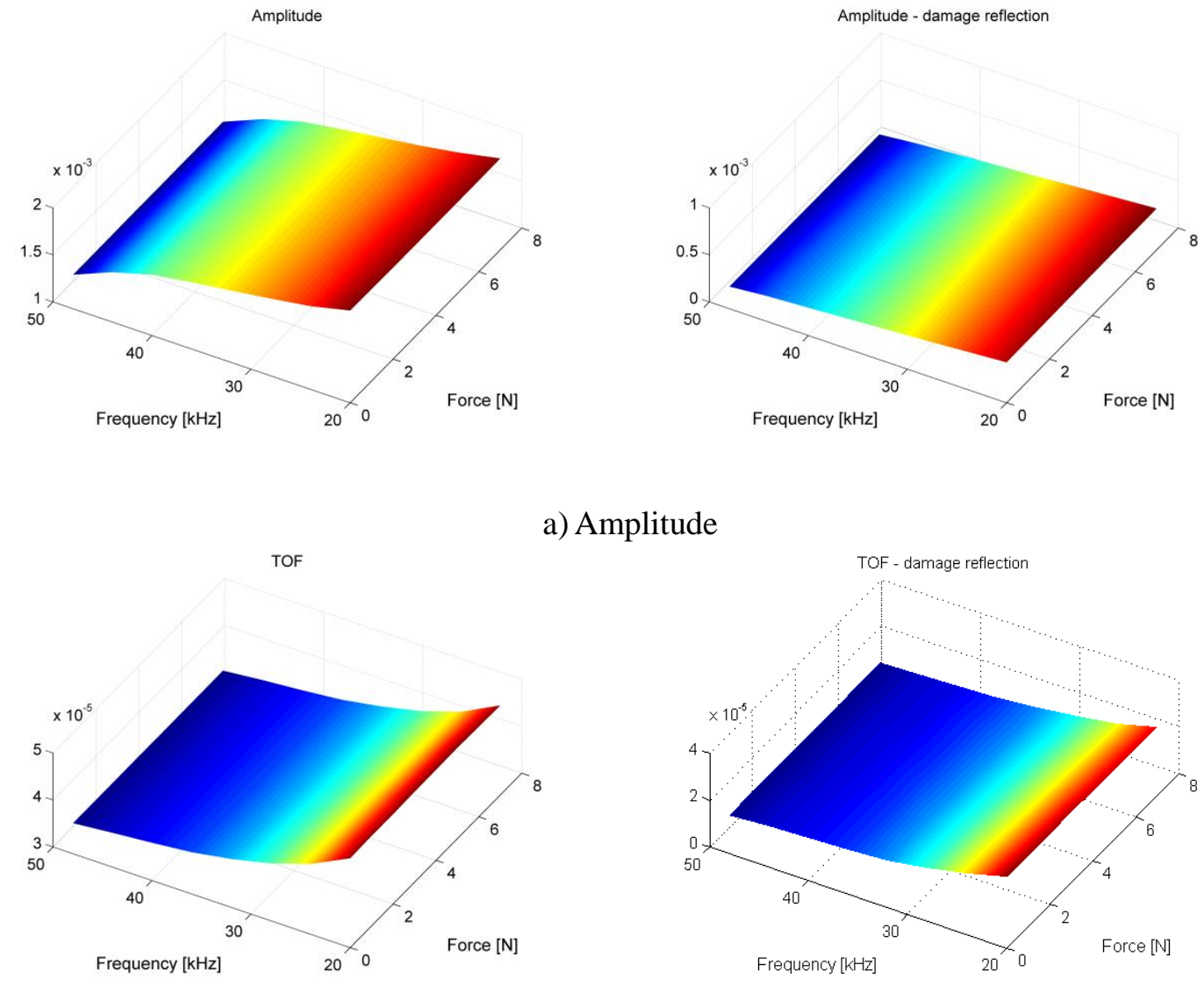

a) Amplitude

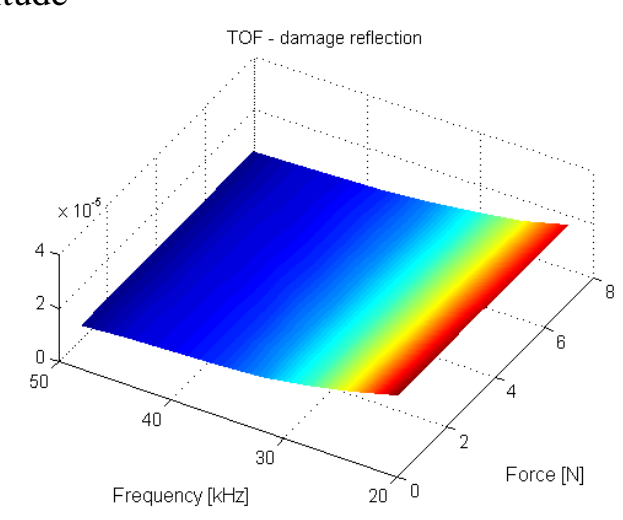

b) Time of Flight (TOF)
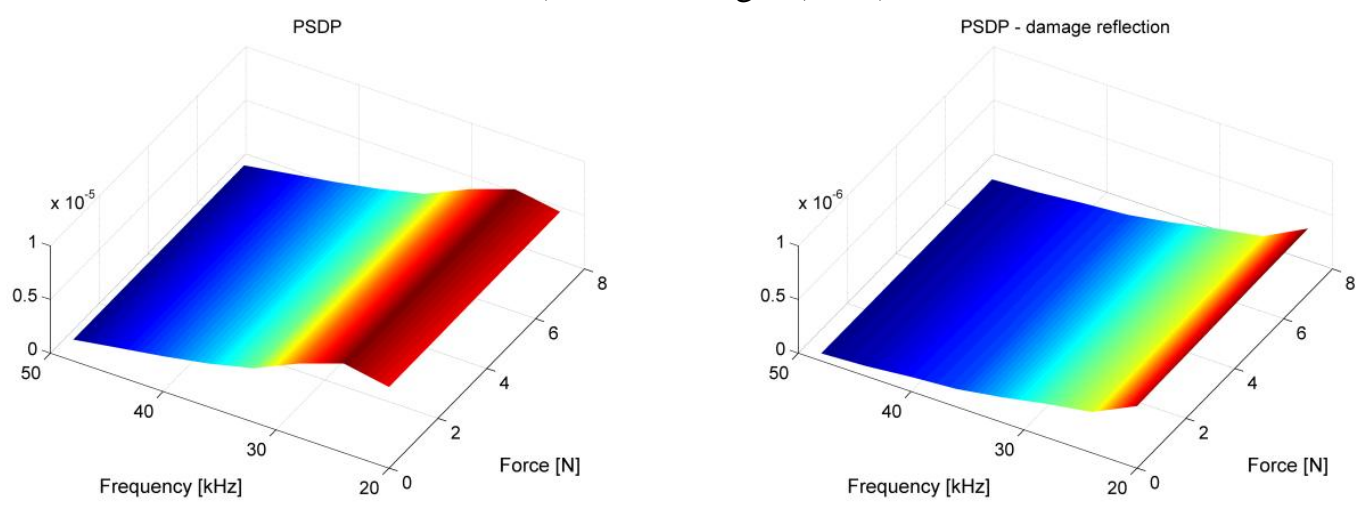

c) Power Spectra Density Peak (PSDP)

Figure 6. Dependence of features on external force. Left column - features for the incoming wave packet, right column - features for the wave packet reflected from the crack. 


\section{CONCLUSIONS}

Algorithm for solving wave propagation problems in composite Timoshenko beams has been developed. It incorporates many effects such as external loading, temperature change and moisture degradation as well as transverse crack. Several features of propagating waves has been analysed in order to find distinction between these effects. It can be concluded that in order to estimate crack depth properly it is necessary to compensate effects of temperature and moisture change in composite structure. Wave propagation is also affected by external loading but in the case considered the loading effect has much less importance than thermal and hygrothermal degradation. Features analysis performed would be used in future for selection of input features for damage identification methods based on machine learning algorithms.

\section{Acknowledgements}

Pawel Kudela would like to gratefully acknowledge the Foundation for Polish Science for the support in his research in the frame of KOLUMB scholarship.

Wieslaw Ostachowicz would like to gratefully acknowledge the support provided by project MONIT - Monitoring of technical state of construction and evaluation of its lifespan (ref. no. POIG.01.01.02-00-013/08).

\section{REFERENCES}

[1] Blaise E., F.K. Chang, "Built-in diagnostics for debonding in sandwich structures under extreme temperatures". Proceedings of the 3rd International Workshop on SHM, 154163, Stanford, California, USA, 2001.

[2] Lu Y., Michaels J.E., "A methodology for structural health monitoring with diffuse ultrasonic waves in the presence of temperature variations". Ultrasonics. 43, 717-731, 2005.

[3] Konstantinidis G., Wilcox P.D., Drinkwater B.W. „An Investigation Into the Temperature Stability of a Guided Wave Structural Health Monitoring System Using Permanently Attached Sensors". IEEE Sens. J. 7(5), 905-912, 2007.

[4] Roy S., Lonkar, K., Janapati, V., Chang, F.-K., "Physics Based Temperature Compensation Strategy for Structural Health Monitoring". Proceedings of the 8th International Workshop on SHM, Stanford University, USA, 1, 1139-1149, 2011.

[5] Ha S., Lonkar, K., Mittal, A., Chang, F.-K., "Adhesive Layer Effects on PZT-induced Lamb Waves at Elevated Temperatures". SHM J., 26, 247-256, 2010.

[6] Kudela P., Ostachowicz W., Zak A., "Influence of temperature fields on wave propagation in composite plates". Key Engineering Materials, 347, 537-542, 2007.

[7] Vinson J.R., Sierakowski R.L., "Behaviour of Structures Composed of Composite Materials", Martinus Nijhoff, Dorchester, 1989.

[8] Abdel-Magdid B., Ziaee S., Gass K., Schneider M.,"The combined effects of load, moisture and temperature on the properties of E-glass/epoxy composites". Compos. Struct., 71, 320-326, 2005.

[9] Tsai Ch.L., Wang Ch.H., Chang J.-J., Yeih W., "Tracking hygrothermal strains of 
carbon/epoxy composite under varying temperature and humidity". J Compos. Mater. 42(16), 1597-1618, 2008.

[10] Sai Ram K.S., Sinha P.K, "Hygrothermal effects on the free vibration of laminated composite plates", J. Sound Vib., 158(1), 133-148, 1992.

[11] Chen F., Wilcox P. D., "The effect of load on guided wave propagation". Ultrasonics, 47(1-4), 645-649, 2007.

[12] Hayashi T., Song W.-J., Rose J. L., "Guided wave dispersion curves for a bar with an arbitrary cross-section, a rod and rail example". Ultrasonics, 41, 175-183, 2003.

[13] Lematre M., Feuillard G., Clézio E. L., Lethiecq M., "Modeling of the influence of a prestress gradient on guided wave propagation in piezoelectric structures". J Acoust. Soc. Am., 120(4), 1964-1975, 2006.

[14] Chakraborty A., "The effect of surface stress on the propagation of Lamb waves". Ultrasonics, 50, 645-649, 2010.

[15] Karadeniz Z.H., Kumlutas D., "A numerical study on the coefficients of thermal expansion of fibre reinforced composite materials". Compos. Struct. 78, 1-10, 2007.

[16] Ostachowicz W, Kudela P., Krawczuk M., Żak A. "Guided Waves in Structures for SHM: The Time-domain Spectral Element Method”, Wiley, 2012.

[17] Tada H., Paris P.C., Irwin G.R., "The Stress Analysis of Cracks Handbook", Del Research Corporation, Hellertown, PA, 1973. 